\title{
Ground state of classical bilayer Wigner crystals
}

\author{
Ladislav ŠAmaj ${ }^{1,2}$ AND Emmanuel TrizaC ${ }^{2}$
}

1 Institute of Physics, Slovak Academy of Sciences, Bratislava, Slovakia

2 Université Paris-Sud, Laboratoire de Physique Théorique et Modèles Statistiques, UMR CNRS 8626, 91405 Orsay, N France.

PACS $64.70 . \mathrm{kp}$ - Ionic crystals

PACS 68.65.Ac - Multilayers

PACS 73.20.-r - Electron states at surfaces and interfaces

\begin{abstract}
We study the ground state structure of electronic-like bilayers, where different phases compete upon changing the inter-layer separation or particle density. New series representations with exceptional convergence properties are derived for the exact Coulombic energies under scrutiny. The complete phase transition scenario -including critical phenomena- can subsequently be worked out in detail, thereby unifying a rather scattered or contradictory body of literature, hitherto plagued by the inaccuracies inherent to long range interaction potentials.
\end{abstract}

The prediction by Wigner that strongly correlated charge carriers in a uniform compensating background could crystallize [1, was first realized experimentally with electrons at the surface of liquid Helium [2, which form a two-dimensional structure. Since then, the study of low dimensional electronic systems has shown no abating and in particular, the bilayer geometry singles out. It appears significantly richer than its monolayer counterpart and has been investigated in different settings : GaAs quantum wells [3] or other semiconductors [4, quantum dots [5], graphene [6], boron nitride 7], laser-cooled trapped ion plasmas 8], dusty plasmas [9] and colloids [10,11. In light of these applications, it is essential to understand the ground state features of Coulombic bilayers, start- ing with the classical limit. This problem has received significant attention in the last 20 years, as such 12 or supplemented with finite temperature analysis [17 19]. In addition, ground state ordering impinges on strongcoupling expansions describing counterintuitive yet ubiquitous electrostatic phenomena, such as like-charge attraction [20, 23] or charge reversal [24,25]. This body of work has revealed the main features of ground state structure, but there exist surprising discrepancies in the literature, especially on the respective domains of existence of the different phases possible. The reason lies in the long range nature of Coulombic interactions, a common bane for such analysis. Our goal here is to resolve existing controversies, precisely locate all phases and discuss the critical behavior associated. All results reported are exact; they are

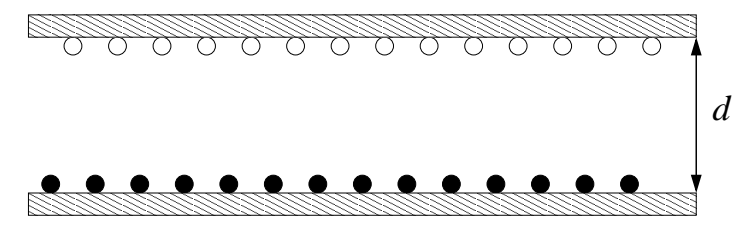

Fig. 1: Side view of the two parallel plates at a separation $d$. From their common uniform surface charge density $-\sigma q$, we define the dimensionless distance as $\eta=d \sqrt{\sigma}$. Ions, which bear charge $q$, are shown as black or white disks for visual ease, but they are point-like in the present study.

obtained from new series representations of lattice sums for Coulomb law.

We consider an ensemble of identical classical point charges $q$, interacting through a $1 / r$ pair potential, and confined between two symmetric parallel charged walls. These boundaries both bear a uniform surface charge of density $-\sigma q$, so that global electroneutrality holds. At finite temperature $T$, the charges do populate the interior of the slab. For $T=0$ though, the charges evenly condense on the opposing walls, thereby forming a bilayer ground state [26, 27] the structure of which depends on a single dimensionless parameter $\eta=d \sqrt{\sigma}$, where $d$ is the interplate distance (see Fig. 1). It is known that five structures can be realized upon increasing $\eta$; they will be referred to following standard terminology, common to the classical [14] and quantum contexts 29]. To begin with, the limits 


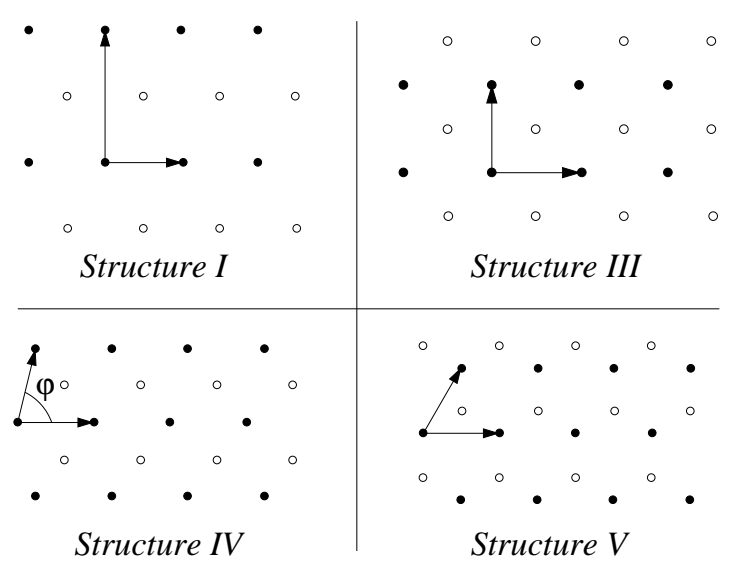

Fig. 2: Schematic representation of the different ground states encountered when the dimensionless distance $\eta$ increases. The open and filled symbols show the locations of ions on the opposite surfaces (see Fig. 1). The arrows are for lattice vectors $\boldsymbol{a}_{1}$ and $\boldsymbol{a}_{2}$, from which we define the aspect ratio $\Delta=\left|\boldsymbol{a}_{2}\right| /\left|\boldsymbol{a}_{1}\right|$ : $\Delta=\sqrt{3}$ and 1 for structures I and III respectively, while structure II interpolates between I and III with $1<\Delta<\sqrt{3}$. For structure IV, the order parameter is the angle $\varphi$ between $\boldsymbol{a}_{1}$ and $\boldsymbol{a}_{2}$. We have $\varphi=\pi / 2$ for structure III whereas $\varphi=\pi / 3$ for structure $\mathrm{V}$, and in general, $\pi / 3 \leq \varphi \leq \pi / 2$. Structures I, III and V are rigid, as opposed to the soft cases II and IV where the unit cell geometry depends on inter-plate separation, through $\Delta$ and $\varphi$, respectively. Note that the shift between the two opposite crystals materialized by open and filled symbols is $\left(\boldsymbol{a}_{1}+\boldsymbol{a}_{2}\right) / 2$ for structures I, II, III, IV but $\left(\boldsymbol{a}_{1}+\boldsymbol{a}_{2}\right) / 3$ for structure V.

of small and large $\eta$ are both straightforward. For $\eta \rightarrow 0$, a genuine two-dimensional one component plasma is produced 20, where the strong mutual repulsion between charges leads to a triangular Wigner crystal [30, the socalled structure I. Conversely, for $\eta \rightarrow \infty$, the two layers decouple and a hexagonal crystal forms on each plate (structure V). These two crystals adopt a staggered configuration, to minimize inter-layer repulsion. For intermediate reduced distances $\eta$, three other structures are met, see Fig. 2. a staggered rectangular lattice (structure II), a staggered square lattice (structure III), and a staggered rhombic lattice (structure IV). Note that while one can evolve continuously through the sequence $\mathrm{I} \rightarrow \mathrm{II} \rightarrow \mathrm{III} \rightarrow$ IV, no continuous deformation allows to create structure $\mathrm{V}$ from one of the others. The transitions between phases will therefore be of different orders, with characteristics and critical exponent (in the continuous cases) that will be worked out explicitly below. A goal of our analysis is to precisely locate the transition points between phases: indeed, a dispersion of about $20 \%$ exists for the hitherto reported threshold $\eta_{\mathrm{IV}}$ between structures IV and V, see [13, 14, 16, 18. In addition, controversial results have been reported for the transition point $\eta_{\mathrm{I}}$ between structures I and II: $\eta_{\mathrm{I}} \simeq 0.006$ from Ewald summation technique [14, $\eta_{\mathrm{I}} \simeq 0.028$ from Monte Carlo simulations [18, whereas lattice sum minimization of Yukawa systems in the un- screened limit hints at $\eta_{\mathrm{I}}=0$ [15], meaning that structure I could possibly only exist at precisely $\eta=0$.

We start by addressing the question whether $\eta_{\mathrm{I}}=0$ or $\neq 0$, and to this end, we compute the energy $E(\Delta, \eta)$ of structure II. For a given layer, the 2D lattice points are indexed by $j \boldsymbol{a}_{1}+k \boldsymbol{a}_{2}$ where $j$ and $k$ are integers and the lattice vectors $\boldsymbol{a}_{1}=a(1,0), \boldsymbol{a}_{2}=a(0, \Delta)$ are shown in bold in Fig. 2. The global electroneutrality requires that $a^{2} \sigma \Delta=1$. The aspect ratio $\Delta$ fulfills $1 \leq \Delta \leq \sqrt{3}$ with $\Delta=\sqrt{3}$ for structure I and $\Delta=1$ for the (square) structure III. The dielectric constant of the medium is set to unity for the sake of simplicity, and the total energy per ion $E(\Delta, \eta)$ is written as the sum of intra- and interlayers contributions. We first restrict ourselves to a disk of finite radius $R$ around a given reference ion located at $(0,0)$. Considering ion-ion and ion-plate interactions, we have the intra-layer energy

$$
E_{\text {intra }}=\frac{q^{2}}{2 a} \sum_{\substack{j, k \\(j, k) \neq(0,0)}} \frac{1}{\sqrt{j^{2}+k^{2} \Delta^{2}}}-\frac{\sigma q^{2}}{2} \int_{0}^{R} d \mathbf{r} \frac{1}{|\mathbf{r}|}
$$

with the restriction $j^{2}+k^{2} \Delta^{2} \leq(R / a)^{2}$. It is expedient and common procedure [19,31] to use the gamma identity $(\pi / z)^{1 / 2}=\int_{0}^{\infty} t^{-1 / 2} \exp (-z t) d t$ valid for $z>0$, which provides us with a simple expression where the limit $R \rightarrow$ $\infty$ can be readily taken:

$$
\begin{array}{r}
\frac{\sqrt{\pi} E_{\text {intra }}}{q^{2} \sqrt{\sigma}}=\frac{1}{2 a \sqrt{\sigma}} \int_{0}^{\infty} \frac{d t}{\sqrt{t}}\left[\sum_{j, k} e^{-t j^{2}} e^{-t k^{2} \Delta^{2}}-1-\frac{\pi}{t \Delta}\right] \\
=\frac{1}{2} \int_{0}^{\infty} \frac{d t}{\sqrt{t}}\left[\sum_{j, k} e^{-t j^{2} / \Delta} e^{-t k^{2} \Delta}-1-\frac{\pi}{t}\right], \\
=\int_{0}^{\pi} d t \frac{\pi}{t^{3 / 2}}\left[\sum_{j, k \neq(0,0)} e^{-(\pi j)^{2} /(\Delta t)} e^{-(\pi k)^{2} \Delta / t}\right]-2 \sqrt{\pi} .
\end{array}
$$

Here, the second line is obtained from the substitution $t \Delta \rightarrow t$ and the condition $a^{2} \sigma \Delta=1$; the third line stems from considering separately the domains $t \in[0, \pi]$ and $t \in[\pi, \infty]$ in the integral, substituting $\pi^{2} / t \rightarrow t$ and subsequently using Poisson summation formula

$$
\sum_{j=-\infty}^{\infty} e^{-(j+\phi)^{2} t}=\sqrt{\frac{\pi}{t}} \sum_{j=-\infty}^{\infty} e^{2 \pi i j \phi} e^{-(\pi j)^{2} / t} .
$$

The inter-layer energy contribution $E_{\text {inter }}$ is amenable to a similar treatment [32, and the last step of the procedure consists in introducing the function

$$
z_{\nu}(x, y)=\int_{0}^{1 / \pi} \frac{d t}{t^{\nu}} e^{-x t} e^{-y / t} \quad \text { for } y>0 .
$$

We finally end up with the series representation for the total energy $E(\Delta, \eta)=E_{\text {intra }}+E_{\text {inter: }}$ The function 


$$
\begin{aligned}
\frac{E(\Delta, \eta) \sqrt{\pi}}{q^{2} \sqrt{\sigma}}= & 2 \sum_{j=1}^{\infty}\left[z_{3 / 2}\left(0, j^{2} / \Delta\right)+z_{3 / 2}\left(0, j^{2} \Delta\right)\right]+4 \sum_{j, k=1}^{\infty} z_{3 / 2}\left(0, j^{2} / \Delta+k^{2} \Delta\right) \\
& +\sum_{j=1}^{\infty}(-1)^{j}\left[z_{3 / 2}\left((\pi \eta)^{2}, j^{2} / \Delta\right)+z_{3 / 2}\left((\pi \eta)^{2}, j^{2} \Delta\right)\right]+2 \sum_{j, k=1}^{\infty}(-1)^{j}(-1)^{k} z_{3 / 2}\left((\pi \eta)^{2}, j^{2} / \Delta+k^{2} \Delta\right) \\
& +2 \sum_{j, k=1}^{\infty} z_{3 / 2}\left(0, \eta^{2}+(j-1 / 2)^{2} / \Delta+(k-1 / 2)^{2} \Delta\right)-2 \sqrt{\pi}-\frac{\pi}{2} z_{1 / 2}\left(0, \eta^{2}\right) .
\end{aligned}
$$

$z_{\nu}$ generalizes to two-layer problems the so-called Misra function 33 used extensively in single-layer lattice summation [34, 35]. Our use of (5) will be three-pronged: it allows to show analytically that $\eta_{\mathrm{I}}=0$, to calculate explicitly the singular behavior near critical points and is moreover particularly suited for numerical evaluations. From an operational point of view, the series (5) is indeed endowed with remarkable properties: it is free of singular terms, and importantly, converges extremely quickly. The error made upon truncating the series in the energy expression (5) at order $j=k=M$ behaves like $\exp \left(-c M^{2}\right) / M$, where $c$ is a constant of order unity. We first document the convergence property on the single-layer case of structure I, for which the exact energy is $E(\sqrt{3}, 0) /\left(q^{2} \sqrt{2 \sigma}\right)=-1.96051578931989165 \ldots$, which is directly the Madelung constant of the 2D hexagonal Wigner crystal. Cutting the series (5) at $M$, we obtain the exact value with a precision of 2,5,10,17 digits with notably small cutoffs $M=1,2,3,4$ respectively. This makes numerical calculations extremely fast and efficient on any workstation. A similar accuracy is met for all other structures and parameter values reported here, and all numerical results quoted below have been obtained with the cutoff $M=5$.

We now turn our attention to the threshold $\eta_{\mathrm{I}}$ which defines the stability window of structure I. For a given distance $\eta$, we proceed by calculating the Taylor expansion of (5) in the small parameter $\epsilon=\sqrt{3}-\Delta$, which yields

$$
\frac{E(\sqrt{3}-\epsilon, \eta)}{q^{2} \sqrt{2 \sigma}}=\frac{E(\sqrt{3}, \eta)}{q^{2} \sqrt{2 \sigma}}+f_{1}(\eta) \epsilon+f_{2}(\eta) \epsilon^{2}+\mathcal{O}\left(\epsilon^{3}\right)
$$

where and the function $f_{2}$ is also explicitly known 32 . To investigate the stability of structure I, it is sufficient to study the sign of $f_{1}$, which is worked out from a Taylor expansion for small $\eta$. The first two derivatives of this function $f_{1}$ vanish at $\eta=0$ and we have $f_{1}(\eta)=-0.5833059875 \ldots \eta^{2}+\mathcal{O}\left(\eta^{4}\right)$, hence an energy gain upon increasing $\epsilon$ as compared to the $\epsilon=0$ case (structure I). This implies that $\eta_{\mathrm{I}}=0$ : at finite but small distances $\eta$, the optimal phase is not structure I. To obtain the optimal value of $\epsilon$ selected and that we denote $\epsilon^{*}$, we further Taylor expand $f_{2}(\eta)$ which yields $f_{2}(\eta)=0.0408440789 \ldots+\mathcal{O}\left(\eta^{2}\right)$. As a consequence,

$$
\sqrt{3}-\Delta^{*} \equiv \epsilon^{*}=-\frac{f_{1}(\eta)}{2 f_{2}(\eta)}=7.14064 \ldots \eta^{2}+\mathcal{O}\left(\eta^{4}\right)
$$

which entails that the energy change scales like $\eta^{4}$. For the thresholds $\eta_{\text {I }}$ reported in Refs. [14, 18, a relative accuracy of $10^{-9}$ was therefore required to answer the finite or vanishing $\eta_{\mathrm{I}}$ question. The accuracy of our findings is

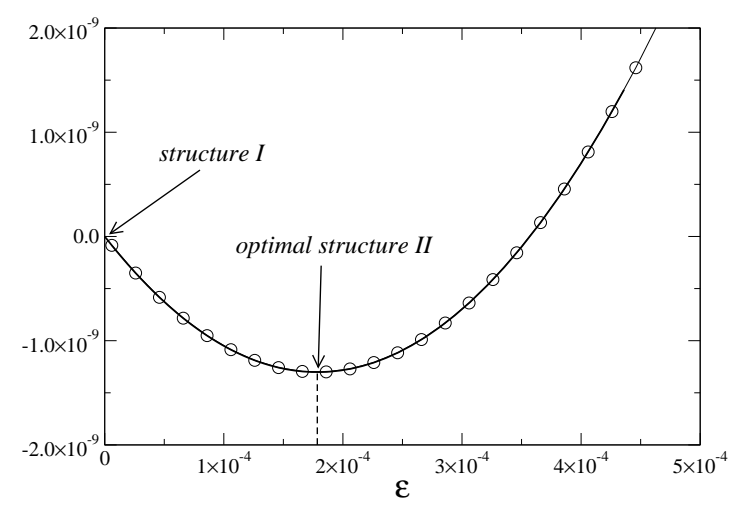

Fig. 3: The difference between the dimensionless energies $[E(\Delta, \eta)-E(\sqrt{3}, \eta)] /\left(q^{2} \sqrt{2 \sigma}\right)$ versus $\epsilon=\sqrt{3}-\Delta$, for $\eta=$ $5.10^{-3}$. The analytical formula (6) with the Taylor expansions of $f_{1}$ and $f_{2}$ given in the text is shown by the continuous line. It is compared to the numerical evaluation of the series (5) with a cutoff $M=5$ (symbols). The optimal $\epsilon$ value following from the prediction (8) is shown by the dashed vertical line.

The above analysis shows that the evolution from structure I to structure II is not a phase transition in the common sense. The situation differs between structures II and III. To inspect the corresponding transition, we note that $E(\Delta, \eta)$ enjoys the symmetry $\Delta \rightarrow \Delta^{-1}$, as is clear from Fig. 2 where a global rotation of $\pi / 2$ does not affect the energy but interchanges lattice vectors $\boldsymbol{a}_{1}$ and $\boldsymbol{a}_{2}$. The value $\Delta=1$ characterizing structure III is therefore a self-dual point, and it will now be convenient to parameterize the aspect ratio as $\Delta=\exp (\epsilon)$. All expressions will then be even in $\epsilon$. The expansion of $E\left(e^{\epsilon}, \eta\right)$ in small $\epsilon$-deviations yields

$$
\frac{E\left(e^{\epsilon}, \eta\right)}{q^{2} \sqrt{2 \sigma}}=\frac{E(1, \eta)}{q^{2} \sqrt{2 \sigma}}+g_{2}(\eta) \epsilon^{2}+g_{4}(\eta) \epsilon^{4}+\mathcal{O}\left(\epsilon^{6}\right),
$$




$$
\begin{aligned}
f_{1}(\eta) & =\frac{1}{2^{3 / 2} \sqrt{\pi}}\left\{4 \sum_{j=1}^{\infty} j^{2}\left[z_{5 / 2}\left(0, j^{2} \sqrt{3}\right)-\frac{1}{3} z_{5 / 2}\left(0, j^{2} / \sqrt{3}\right)\right]+8 \sum_{j, k=1}^{\infty}\left(k^{2}-\frac{j^{3}}{3}\right) z_{5 / 2}\left(0, j^{2} / \sqrt{3}+k^{2} \sqrt{3}\right)\right. \\
& +2 \sum_{j=1}^{\infty}(-1)^{j} j^{2}\left[z_{5 / 2}\left((\pi \eta)^{2}, j^{2} \sqrt{3}\right)-\frac{1}{3} z_{5 / 2}\left((\pi \eta)^{2}, j^{2} / \sqrt{3}\right)\right]+4 \sum_{j, k=1}^{\infty}(-1)^{j+k}\left[k^{2}-\frac{j^{2}}{3}\right] z_{5 / 2}\left((\pi \eta)^{2}, \frac{j^{2}}{\sqrt{3}}+k^{2} \sqrt{3}\right) \\
& \left.+4 \sum_{j, k=1}^{\infty}\left[\left(k-\frac{1}{2}\right)^{2}-\frac{1}{3}\left(j-\frac{1}{2}\right)^{2}\right] z_{5 / 2}\left(0, \eta^{2}+(j-1 / 2)^{2} / \sqrt{3}+(k-1 / 2)^{2} \sqrt{3}\right)\right\}
\end{aligned}
$$

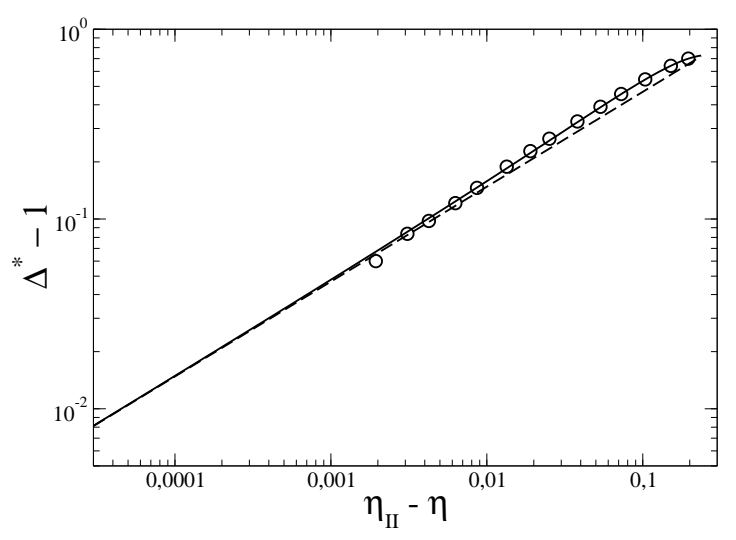

Fig. 4: The transition II $\rightarrow$ III: Test of the analytical asymptotic relation (10) (dashed line) against numerical minimization of the energy (5) (solid curve), in a log-log scale. The numerical data of Ref. 14] are shown by the circles.

where $g_{2}$ and $g_{4}$ are explicitly known [32, in a series form that is very reminiscent of Eq. (77). The bilayer energy appears in a standard Ginzburg-Landau form [36, but it should be emphasized that at variance with mean-field arguments usually underlying such approaches, our expression is exact. The critical point $\eta_{\text {II }}$ sought for is the root of $g_{2}(\eta)=0$, which gives $\eta_{\mathrm{II}}=0.2627602682 \ldots$ It appears here that the thresholds reported in earlier works were accurate: 0.27 [12, 0.262 [14, 0.27 [16] and 0.28 [18. Proceedings along similar lines as for the I $\rightarrow$ II crossover, we Taylor expand $g_{2}(\eta)$ and $g_{4}(\eta)$ to leading order around $\eta_{\mathrm{II}}$. The former behaves like $\left(\eta-\eta_{\mathrm{II}}\right)$ while the latter is constant, a prototypical scenario for a continuous phase transition with critical index $\beta=1 / 2$ [36]. Specifically, we get

$$
\Delta^{*}-1 \simeq \epsilon^{*}=\left(-\frac{g_{2}(\eta)}{2 g_{4}(\eta)}\right)^{1 / 2} \simeq 1.48031 \sqrt{\eta_{\mathrm{II}}-\eta} .
$$

This expression applies for $\eta \leq \eta_{\mathrm{II}}$, in the stability domain of structure II, and is in excellent agreement with our numerical calculations from Eq. (5), see Fig. 4.

The task remaining is to find the series representations for structures IV and V. We first address structure IV. Implementation of the procedure that led to the series (5) becomes possible once the distance between a reference ion and an arbitrary ion located on the same layer at $\mathbf{r}(j, k)=$ $j \boldsymbol{a}_{1}+k \boldsymbol{a}_{2}$ is expressed as

$$
\begin{aligned}
& |\mathbf{r}(j, k)|^{2}=a^{2}\left(j^{2}+k^{2}+2 j k \cos \varphi\right) \\
& =a^{2}\left[(j+k)^{2} \cos ^{2}(\varphi / 2)+(j-k)^{2} \sin ^{2}(\varphi / 2)\right]
\end{aligned}
$$

The latter "diagonalized" form in terms of indices, provides the starting point to write the intra-layer Coulomb energy (summing $1 / \sqrt{|\mathbf{r}(j, k)|^{2}}$ ), and suggests to introduce new indices $n$ and $m$ : if $j+k$ is even, we define $n=(j+k) / 2$ and $m=(j-k) / 2$. If $j+k$ is odd, we introduce indices $n=(j+k+1) / 2$ and $n=(j-k+1) / 2$. Likewise for the inter-layer interactions, taking due account of the shift $\left(\boldsymbol{a}_{1}+\boldsymbol{a}_{2}\right) / 2$ between opposite layers. Building on the gamma identity and Poisson summation formula, the series form for the energy $E_{\mathrm{IV}}$ ensues 32. This energy depends on the angle $\varphi$ and of course on the distance $\eta$. For our purposes, rather than the lengthy explicit form, it is sufficient to report the Landau-like expansion of $E_{\mathrm{IV}}$ in the vicinity of $\varphi=\pi / 2$. A convenient expansion parameter is $\epsilon$ such that $\exp (\epsilon)=\tan (\varphi / 2)$, and the invariance $\varphi \rightarrow \pi-\varphi$ makes $E_{\mathrm{IV}}$ an even function of $\epsilon$. In the small $\epsilon$ region of interest associated to the vicinity of $\pi / 2$ for $\varphi$, we obtain an expansion up to order $\epsilon^{4}$ of the same form as (91). This teaches us that structure III is unstable for $\eta>\eta_{\text {III }}=0.6214809246 \ldots$. to be compared to the thresholds 0.61 [13], 0.622 [14, 0.62 [16], 0.59 [18] while structure IV was not considered in [12]. We furthermore again obtain a second order phase transition with critical index $1 / 2$ and explicit order parameter close to the transition point

$$
\epsilon^{*} \simeq \frac{\pi}{2}-\varphi^{*} \simeq 1.24494 \sqrt{\eta-\eta_{\mathrm{III}}},
$$

in excellent agreement with our numerical data.

The transition IV $\rightarrow \mathrm{V}$ is discontinuous, which made its characterization more elusive in previous publications. Our method, though, is easily adapted to the geometry of structure V. The series representation for $E_{\mathrm{V}}(\eta)$ should be compared to $E_{\mathrm{IV}}\left(\varphi^{*}, \eta\right)$ evaluated for the optimal distortion angle $\varphi^{*}(\eta)$. Requiring that $E_{\mathrm{V}}(\eta)=E_{\mathrm{IV}}\left(\varphi^{*}, \eta\right)$, we obtain the last $\eta$-threshold that was remaining to be specified: $\eta_{\mathrm{IV}}=0.73242 \ldots$ Previous investigations gave 0.75 [13, 0.732 [14, 0.87 [16 and 0.70 [18. For $\eta>\eta_{\mathrm{IV}}$, structure $\mathrm{V}$ is energetically favorable. As a by-product of our analysis, we report the large distance behavior of the 
interplate pressure $P=-2 \sigma \partial E_{\mathrm{V}} / \partial d$ :

$$
P=-6 \pi(\sigma q)^{2} \exp \left(-\frac{4 \pi}{\sqrt{2} 3^{1 / 4}} \eta\right)
$$

in agreement with [14] but at variance with [13,22].

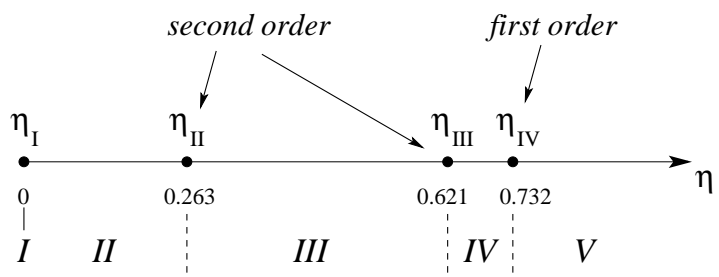

Fig. 5: Sequence of structures encountered as a function of reduced inter-plate separation $\eta$. The values reported for the different stability thresholds are rounded to the third digit.

To summarize, we have derived series representations for the different Coulomb lattice sums pertaining to the ground state of classical bilayer systems. The derivation, worked out explicitly for the five different structures that were known to compete at vanishing temperature, results from a series of transformation rooted in the general theory of Jacobi $\theta$ functions [32. The resulting series provide the thresholds delimiting the domains of validity of the different phases, that were prone to some fluctuations in previous works. Figure 5 provides an overview of our main findings. We could in particular show that the simple hexagonal structure I can only exist in the limiting case of a vanishing interplate distance, and is preempted by a buckled phase for all $\eta \neq 0$. This is the scenario first reported in Ref. [15], which differs from several other studies that assigned a finite stability window to phase I. Whereas the evolution I $\rightarrow$ II is not a phase transition, we could show that the continuous transitions II $\rightarrow$ III and III $\rightarrow$ IV have critical index $\beta=1 / 2$. In addition, our series representation is endowed with exceptional convergence properties, providing typically more than 10 digits of accuracy when retaining only the first 4 or 5 terms involved. Relinquishing the symmetry between the two plates to address the cases where they bear different surface charges is an interesting venue for future work. This brings the difficulty that local electroneutrality no longer holds at the single-plate level in the ground state 37, except presumably at large separations. Our approach can also be extended to bilayers and multilayers with repulsive Yukawa or inverse-power-law interactions, that deserve attention.

$$
* * *
$$

We would like to thank M. Mazars and C. Texier for useful discussions. The support received from Grants VEGA No. 2/0049/12 and CE-SAS QUTE is acknowledged.

\section{REFERENCES}

[1] E. Wigner, Phys. Rev. 46, 1002 (1934).

[2] C.G. Grimes and G. Adams, Phys. Rev. Lett. 42, 795 (1979).

[3] H. C. Manoharan, Y. W. Suen, M. B. Santos, and M. Shayegan, Phys. Rev. Lett. 77, 1813 (1996); E. Tutuc, M. Shayegan, and D. A. Huse, Phys. Rev. Lett. 93, 036802 (2004).

[4] J. P. Eisenstein and A. H. MacDonald, Nature 432, 691 (2004).

[5] H. Imamura, P. A. Maksym and H. Aoki, Phys. Rev. B 53, 12613 (1996).

[6] A. S. Mayorov, D. C. Elias, M. Mucha-Kruczynski, R. V. Gorbachev, T. Tudorovskiy, A. Zhukov, S. V. Morozov, M. I. Katsnelson, V. I. Falko, A. K. Geim and K. S. Novoselov, Science 6044, 860 (2011).

[7] R. M. Ribeiro and N. M. R. Peres, Phys. Rev. B 83, 235312 (2011).

[8] T. B. Mitchell, J. J. Bollinger, D. H. E. Dubin, X.-P. Huang, W. M. Itano, B. M. Baughman, Science 282, 1290 (1998).

[9] L. Teng and P. Tu, L. I, Phys. Rev. Lett. 90, 245004 (2003).

[10] S. Neser, C. Bechinger, P. Leiderer and T. Palberg, Phys. Rev. Lett. 79, 2348 (1997).

[11] Note though that in the soft matter realm, the charge carriers need to be multivalent in order to reach, at room temperature and in an aqueous dispersion, the strong couplings required for Wigner crystallization [21,24].

[12] V.I. Falko, Phys. Rev. B 49, 7774 (1994).

[13] K. Esfarjani and Y. Kawazoe, J. Phys.: Condens. Matter 77217 (1995).

[14] G. Goldoni and F. M. Peeters, Phys. Rev. B 53, 4591 (1996).

[15] R. Messina and H. Löwen, Phys. Rev. Lett. 91, 146101 (2003); E. C. Oğuz, R. Messina, and H. Löwen, Europhys. Lett. 86, 28002 (2009).

[16] V. Lobaskin and R. R. Netz, Europhys. Lett. 77, 38003 (2007).

[17] I. V. Schweigert, V. A. Schweigert, and F. M. Peeters, Phys. Rev. Lett. 82, 5293 (1999); Phys. Rev. B 60, 14665 (1999).

[18] J. J. Weis, D. Levesque, and S. Jorge, Phys. Rev. B 63, 045308 (2001).

[19] M. Mazars, Phys. Rep. 500, 43 (2011).

[20] Y. Levin, Rep. Prog. Phys. 65, 1577 (2002).

[21] A. Naji, M. Kanduc, R. R. Netz and Rudolf Podgornik, in Understanding Soft Condensed Matter via Modeling and Computation, Eds. W.-B. Hu and A.-C. Shi (World Scientific, Singapore, 2010).

[22] A. W. C. Lau, D. Levine, and P. Pincus, Phys. Rev. Lett. 84, 4116 (2000).

[23] L. Šamaj and E. Trizac, Phys. Rev. Lett. 106, 078301 (2011); Phys. Rev. E 84, 041401 (2011).

[24] A. Y. Grosberg, T. T. Nguyen, and B. I. Shklovskii, Rev. Mod. Phys. 74, 329 (2002).

[25] A. Travesset and D. Vaknin, Europhys. Lett. 74, 181 (2006).

[26] Indeed, Earnshaw theorem [S. Earnshaw, Trans. Camb. Phil. Soc., 7, 97 (1842)] states that a collection of point charges cannot be maintained in stable equilibrium under the action of electrostatic forces alone. Hence, at $T=0$, 
the charges stick to the boundaries, and they do so evenly. The particle surface density on each plate is then $\sigma$, so that electroneutrality holds at the level of each plate. For asymmetric plates, the density of particles may violate neutrality at the plate level, global neutrality being always enforced 37. A similar argument can be found in footnote 27 of Ref. 28.

[27] Clearly, the ground state structure does not depend on the precise value of the confining plates surface charge, as long as they are symmetric objects, thereby producing a vanishing electric field in the interstitial slab [28].

[28] R. Messina, J. Phys.: Condens. Matter 21113102 (2009).

[29] S. Narasimhan and T.-L. Ho Phys. Rev. B 52, 12291 (1995).

[30] L. Bonsall and A. A. Maradudin, Phys. Rev. B 15, 1959 (1977).

[31] S. W. de Leeuw, J. W. Perram and E. R. Smith, Proc. R. Soc. Lond. A, 373, 27 (1980).

[32] L. Šamaj and E. Trizac, in preparation.

[33] R. D. Misra, Proc. Camb. Phil. Soc. 36, 173 (1940); M. Born and R.D. Misra, Proc. Camb. Phil. Soc. 36, 466 (1940).

[34] D. Borwein, J. M. Borwein, R. Shail, and I. J. Zucker, J. Phys. A: Math. Gen. 21, 1519 (1988).

[35] M. J. Bowick, A. Cacciuto, D. R. Nelson, and A. Travesset, Phys. Rev. B 73, 024115 (2006).

[36] L. D. Landau and E. M. Lifshitz, Statistical Physics, Course of Theoretical Physics vol 5, Pergamon Press (1980).

[37] R. Messina, C. Holm, and K. Kremer, Phys. Rev. Lett. 85, 872 (2000). R. Messina, C. Holm and K. Kremer, Europhys. Lett. 51, 461 (2000). 